\title{
The effects of a 6 week dynamic core stability plank exercise program compared to a traditional supine core stability strengthening program on diastasis recti abdominis closure, pain, oswestry disability index (ODI) and pelvic floor disability index scores (PFDI)
}

Lori Maria Walton ${ }^{1,2^{*}}$, Adislen Costa ${ }^{1,2}$, Donna LaVanture ${ }^{1,2}$, Sarah Mcllrath ${ }^{1,2}$ and Brittney Stebbins $s^{1,2}$

*Correspondence: zahramaria7@gmail.com

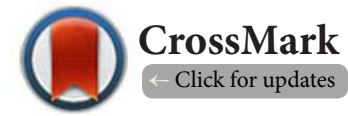

'Department of Physical Therapy, School of Health Sciences, Andrews University, Michigan, USA.

${ }^{2}$ International Academy on Women's Health and Gender Equity Studies, Illinois, USA.

\begin{abstract}
Purpose/Hypothesis: The purpose of this randomized controlled study was to determine if there was a significant difference between a six week traditional treatment program compared to an experimental treatment program consisting of core stabilization plank exercise routine in the closure of diastasis recti abdominis (DRA) and to determine the relationship between DRA measurements, pain, Oswestry Disability Index (ODI), and Pelvic Floor Disability Index scores (PFDI).

Study design: Subjects were recruited in the local community utilizing a purposive sampling technique that considered women (postpartum 3 months to 3 years) between the ages of 18-45. Nine subjects volunteered to be in the study, signed an informed consent, and were randomly selected to be in either in a traditional or experimental group. Each participant completed both pre and post test diagnostic ultrasound and caliper measurements for DRA, health questionnaire, PFDI, and ODI scores.

Materials and methods: A pilot study $(n=5)$ was performed prior to the full study and indicated significant and strong inter-rater and intra-rater reliability for the diagnostic ultrasound DRA measurement at $(r=0.945-0.989$, $\mathrm{p}<0.0005)$. All participants were measured prior to being randomly placed into either the traditional or experimental group. Co-researchers who were in charge of DRA measurement were blinded from the group designation. The traditionalgroup received a supine strengthening program and the experimental group received a dynamic core stabilization program including the addition of plank exercise to approximate the DRA.

Results: Overall, in both groups there was a measured, significant decline in the DRA measurement at the umbilicus $(\mathrm{F}=7.28, \mathrm{p}=0.036)$, but the traditional group showed a slightly greater decline from pre to posttest $(\mathrm{M}($ pre $)=10.97+/-1.96 ; \mathrm{M}($ post $)=6.63+/-1.65)$ compared to the experimental group $(\mathrm{M}($ pre $)=8.75+/-0.87)$ $(\mathrm{M}($ post $)=7.58+/-2.01)$. Disability scores also revealed a significant decline with respect to the ODI for both the traditional group $(\mathrm{Z}=-1.95, \mathrm{p}=0.50)(\mathrm{M}(\mathrm{pre})=5.50+/-5.51)(\mathrm{M}(\mathrm{post})=3.50+/-3.00)$ and the experimental group $(\mathrm{M}($ pre $)=14.40+/-15.39)(\mathrm{M}($ post $)=4.40+/-3.58)$. A significant difference was not found within groups, from pre to post-test, for the overall PFDI scores and its components (UDI and POPDI). However, a significant difference was found, within group, with respect to CRADI scores $(\mathrm{Z}=-2.032, \mathrm{p}=0.042)$. In addition, a significant difference was not found between women who reported participation in pre-natal exercises compared with those who did not participate in pre-natal exercises with respect to baseline DRA measurements (above umbilicus: $\mathrm{Z}=-0.980, \mathrm{p}=0.327$ ), (at umbilicus: $\mathrm{Z}=-0.735, \mathrm{p}=0.462$ ), and (below umbilicus: $\mathrm{Z}=-0.980, \mathrm{p}=0.327$ ). Finally, a negative but non-significant correlation was found between PFDI scores and DRA closure measurements $\left(r=0.605, r^{2}=0.366, p=0.084\right)$.

Conclusions: Both the traditional and experimental group showed significant reduction in DRA measurement from pre to posttest, with the traditional program exhibiting a slightly greater decline from pre to post DRA measurement than the experimental group. These findings suggest that either strengthening program, traditional or experimental, could be effective at reducing DRA measurement in postpartum women. However, this study was limited by a small sample size and would benefit from future research focused on specific exercise prescription progression.
\end{abstract}

Keywords: Diastasis rectus abdominis, diagnostic ultrasound, pelvic rehabilitation 
Walton et al. Physical Therapy and Rehabilitation 2016,

\section{Introduction}

Diastasis rectus abdominis (DRA) is a common health problem in both pregnant and postpartum populations. Benjamin, Van de Water, and Peiris (2014) found in their systematic research that almost all pregnant women experienced DRA during the third trimester and nearly half of women experience it immediately after the child is born because of the stress of child delivery [1]. The primary muscle effected by these changes during pregnancy is the rectus abdominis, which is connected to the lineaalba. The two muscle bellies of the rectus abdominis begin to stretch and lengthen due to the growing fetus. This ongoing process that occurs throughout the pregnancy can result in a separation of the muscles and, potentially, protrusion of the umbilicus (Mota, Pascoal, Sancho, and Bo, 2012) [2]. This process continues throughout the duration of a woman's pregnancy. Due to the stretching of the lineaalba, the two rectus abdominis muscles separate, resulting in DRA. Beer et al., (2009) found, in a study of 150 nulliparous women, that the normal measurements for the linea alba are as follows: "The mean width was 7+/-5 $\mathrm{mm}$ at the xiphoid and $13+/-7 \mathrm{~mm}$ above and $8+/-6 \mathrm{~mm}$ below the umbilicus" [3]. If the lineaalba measurement exceeds these numbers, then a diagnosis of DRA could result.

Once the birthing process is complete, the mother's body will start to readapt and attempt to return to its original state. If the lineaalba does not regain its elastic fibers, numerous health problems can occur as a result. One of the major health complications of DRA is low back pain. Around four out of ten women report persistent LBPP [low back pelvic pain] half a year after delivery" (Parker, Dugan, \& Millar, 2009) [4]. Current studies have shown that DRA presence may contribute to the occurrence and persistence of low back pain in postpartum women. It has been determined that women with a presence of DRA are predisposed to having a higher degree of pain in the abdominal and pelvic region postpartum (Parker, Dugan, \& Millar, 2009) [4].

One way to reduce the presence of DRA is through means of physical activity during pregnancy. A recent study concluded that if pregnant women participate in a strengthening exercise program focused on transverse abdominis contraction with upper and lower limb movement throughout the duration of their nine month term, the presence of DRA is significantly lower than that of women who do not exercise while pregnant (Chiarello, Falzone, McCaslin, Patel, and Ulery, 2005) [6]. Yet, a study conducted by Mogren (2008) found that half the women who participated in the study failed to participate in physical exercise on a regular basis until two and half months after delivery [5].

To the authors' knowledge, there is no evidence specifically explaining the mechanism of how abdominal exercises can reduce DRA, but this continues to be the norm. Isometric contraction when performing a supine abdominal crunch has been shown to reduce DRA as shown in a preliminary casecontrol study, which compared postpartum to nulliparous women. The researchers found that DRA was much greater in the postpartum women than the nulliparous women; however, the distance was reduced in both groups when an isometric contraction was performed as compared to at rest (Pascoal, Dionisio, Cordeiro, \&Mota, 2014) [7]. Other interventions such as abdominal bracing have been utilized to help reduce DRA in postpartum women. The use of the abdominal brace helps provide support to overstretched muscles and ligaments as well as support for the low back region. A study conducted by El-Mekawy, Eldeeb, El-Lythy, and El-Begawy (2013), divided the participants into two groups: one group utilized only an abdominal brace while the other participated in an abdominal strengthening program without the use of the brace [8]. The following exercises were used to help reduce DRA: static abdominal contraction, posterior pelvic tilt, reverse sit up, trunk twist, and reverse trunk twist; however, there was no significant differences between the two groups. The use of an abdominal brace proved to reduce DRA as effectively as the abdominal strengthening program in that study.

A woman's DRA should be expected to reduce in size, in the acute postpartum period. There is not enough evidence to determine how DRA is able to reduce spontaneously after delivery. The authors' assume that DRA may reduce because of relief of stretch on the anterior abdominal wall in the postpartum period, as well as hormonal influences. Throughout the postpartum period, the resolution of DRA has been shown to progress at different rates and in different patterns. External factors may complicate the natural resolution, causing an increased risk of DRA. Examples of external factors would be an increase in physical demands, valsalva maneuver, muscle fatigue, and fetal position (Hsia and Jones, 2000) [9]. According to Gilleard and Brown (1996), one's DRA at eight weeks postpartum has the potential to be a level similar to 22 weeks gestation [10].

The purpose of this study was to determine if a six weekexperimental plank exercise program, as compared to a traditional supine exercise program prescribed by a licensed physical therapist was effective in the closure of DRA and to determine the relationship between DRA closure in the postpartum period, low back pain, and health-related quality of life.

\section{Methods}

\section{Subjects}

This study utilized a purposive sampling of nine postpartum women, ages 18 to 45, recruited from approved local facilities within the region via a flyer displayed to the general public at the front of their facility. These subjects were then randomly placed into either the traditional treatment or experimental treatment group, both requiring pre and post test measurements. Eligibility criteria included: three months to three years postpartum with the presence of diastasis rectus abdominis. Exclusion criteria included: subjects with any heart condition, respiratory condition, including excessive coughing or sneezing, any pelvic or abdominal surgery (except for a Cesarean section), 
any neurologic disease, trauma to bowel or bladder, prior history of physical therapy treatment for pelvic floor issues, and depression.

Research has shown that postpartum women are more likely to have low back and pelvic pain than nulliparous women. The researchers in this study did not use this as a qualification for admission to participate, but it was monitored throughout treatment. Researchers hypothesized that subjects would have low back or pelvic pain due to decreased abdominal stability from the presence of DRA causing more stress to be put on the low back region. Each subject, who volunteered to take part in the study and signed the informed consent, was screened via a health questionnaire, and if they qualified, they were randomly assigned to one of the two treatment groups.

\section{Instrumentation}

\section{Ultrasound imaging machine}

A Diagnostic Ultrasound Imaging (USI) unit was utilized to measure the DRA. According to Mota, Pascoal, Sancho, and Bo (2012) [2] good reliability was reported in measuring DRA at rest with the use of USI (ICC values: $0.74-0.90$ ). The specific reliability measurements were reported at: "strong at $2 \mathrm{~cm}$ above the umbilicus (ICC of 0.87 (95\% Cl: $0.73,0.94)$ and "good to strong" at $2 \mathrm{~cm}$ below (ICC of 0.78 (95\% Cl: 0.56, 0.90) at rest" [2]. Also, according to Mendes et al., USI was shown to be a valid tool to use in measuring DRA at and above the umbilicus level. The following is a list of significance related values obtained in the following order: $6 \mathrm{~cm}$ above $(p=0.1292)$, $3 \mathrm{~cm}$ above $(p=0.4562)$, and at umbilicus $(p=0.3015)^{\prime \prime}[11]$.

\section{Digital nylon calipers}

In addition, the researchers used digital nylon calipers as a measurement tool because they have also been shown to be reliable and valid, as well as a more accessible tool. Barbosa, Moreira de Sa, and Coca Velarde's (2013) comparison of the reliability of digital nylon calipers when compared to USI revealed an "agreement between the means of measurements at all levels" [12]. It demonstrated a moderate correlation $(r=0.5)$ in the first levels $(3,6$, and $9 \mathrm{~cm})$ [12]. To evaluate the concurrent validity of digital nylon calipers in comparison to USI, Chiarello and McAuley's study (2013) showed that "above the umbilicus, there was no statistically significant difference ( $p>0.05$ and ICC of 0.79 ) between the two tools at rest" [13]. However, measurements taken via the caliper below the umbilicus were not comparable (significantly larger $(p<0.0001)$ to USI) [13].

\section{Oswestry disability index}

Smeets et al., (2010) determined the ODI demonstrated "good to strong internal consistency (Cronbach's $\alpha=0.71-0.87$ ) and test-retest reliability (intraclass correlation coefficient 0.84 , $95 \%$ confidence interval 0.73-0.91)" [14]. It is suggested that "a 30\% score improvement" (Smeets et al., 2011) [14] or a "change of at least 10.5 points" (Davidson and Keating 2002)
[15] should be seen to be "90\% confident that real change has occurred" [15]. Since the ODI had demonstrated both reliability and validity in previous studies the researchers believed the ODI was an effective measurement tool for the purpose of this study.

\section{Pelvic floor distress index}

Geller et al., (2007) determined there was not a significant difference between telephone administration and the written administration $(p=0.36)$ [17]. The mean telephone score was $9.0 \pm 19.3$ while the mean written score was $9.8 \pm 17.7$ which showed a high correlation $(r=0.96)$ between the scores of the two different administration modes [17]. A high correlation was found "within the 3 subscales of each questionnaire, $95 \%$ confidence intervals of the difference in scores between written and telephone versions were also calculated" (Geller et al., 2007) [17]. The subscales of the PFDI consist of (1) the Urinary Distress Inventory (UDI), the (2) Colorectal -Anal Distress Inventory (CRADI), and the (3) Pelvic Organ Prolapse Distress Inventory (POPDI). Barber, Walters, and Bump (2005) found that the long-scale of the PFDI correlates with its short-scales, including the UDI ( $r=0.86)$, CRADI $(r=0.93)$, and POPDI ( $r=0.92)$ [18]. The PFDI and its subscales were used in the study to obtain measurements of pelvic floor issues such as urinary incontinence.

\section{Procedures}

This research study received approval in December 2014 from Andrews University IRB and permission for the use of the physical therapy facility was also obtained from the physical therapy program prior to the start of the research study process.

\section{Phase I}

Two of the co-researchers, in collaboration with the primary investigator,conducted a pilot study to assess the intra-tester and inter-tester reliability for diagnostic ultrasound and caliper. Concurrent criterion validity was also checked at this time. The co-researchers were also designated to measure DRA during the actual study. The procedure for this pilot study closely resembled the actual measurement procedure that was followed in the actual study. It consisted of recruitment of five female subjects who were instructed to lay at rest in a supine position and then perform a small crunch a total of 18 times. Each subject was measured for DRA using both the USI and caliper measuring tools, three measurements, by each of the researchers at three locations: $4.5 \mathrm{~cm}$ above and below the umbilicus, as well as, at the level of the umbilicus. The data were collected by a third and fourth researcher to provide a mechanism for reduction of bias in the data collection process.

\section{Phase II}

After completing the consent form, each subject completed the ODI and PFDI questionnaires. Participants were randomly 
Walton et al. Physical Therapy and Rehabilitation 2016,

divided into two groups, the traditional exercise group versus the experimental exercise group. Subjects were divided randomly into either the traditional or experimental treatment group. Initial measurements of each subject's DRA were taken at rest and with contraction for both the caliper and diagnostic ultrasound. Measurement locations were the same as in the pilot study. The remaining two researchers had access to each subject's contact information and followed up by calling them every two weeks for a total of six weeks. The two researchers who called subjects were blinded to the participant's DRA measurements. Furthermore, the two researchers, who conducted measurements, were blinded to exercise group assignment of each subject.

After initial measurements were taken and recorded, two researchers instructed the subjects on their respective home exercise program. The traditional treatment group completed the following exercises with the use of abdominal bracing: abdominal crunch, posterior pelvic tilt, kegels, and Russian twist. The experimental treatment group completed the following exercises with the use of abdominal bracing: plank, posterior pelvic tilt, kegels, and Russian twist. Abdominal bracing for this research study was defined as using a large towel or sheet secured around the abdominal section for each patient. The rationale for the abdominal bracing was to help increase the closure rate of the DRA.

The supine abdominal crunch has been the standard treatment for DRA reduction. Numerous studies define an abdominal crunch as lifting one's head and shoulders off the table $[6,8,19]$. A posterior pelvic tilt can be performed in a supine or standing position. For this study, the subjects were instructed to complete a posterior pelvic tilt in a supine position. A posterior pelvic tilt has been defined as rolling the pelvic bones simultaneously upward and backward $[6,8,19]$. A kegel, a standard women's health exercise, has been defined as contracting and relaxing the pelvic floor muscles $[6,8,19]$. A Russian twist movement has been defined as contracting the abdominal muscles, with the body in an abdominal crunch position, while simultaneously performing a twisting trunk motion [8]. Lastly, a plank abdominal exercise has been defined as an abdominal exercise that works both the upper and lower body while also strengthening the postural muscles as well. To complete this exercise, one must maintain a prone position while stabilizing themselves on their forearms and feet or knees [19]. To the authors' knowledge, no known evidence has specifically explained the rationale as to why the plank exercise has not been used for DRA reduction. The authors infer that plank exercises may not be prescribed for DRA closure due to their complexity and increased overall body strength to complete with proper form and technique.

Three sets of ten repetitions were done for each exercise, except for the plank which was held for 10 seconds for each repetition. Each subject was given an exercise booklet with instructions and pictures of each exercise as well as a log sheet to record their exercises. As the weeks progressed, subjects were encouraged to increase their repetitions to their personal fatigue level. Researchers defined fatigue level as the moment the subject began to compensate proper form for the respective exercise, which was demonstrated and critiqued at the initial instruction session. The subjects were instructed to complete their respective programs three times a week for a total of six weeks. Researchers contacted each subject via telephone on the second, fourth, and sixth week to reassess how each was completing their home exercise program. Researchers encouraged compliance by emphasizing proper form and answering questions from the subjects.

On the sixth week, subjects were instructed to return for final measurements and questionnaire completion for posttest data collection for comparison. Post measurements of participant's DRA were taken by the same two researchers, in the same locations. The same two measurement tools were utilized for post-test measurement of DRA.

\section{Data analysis}

All data were compiled into SPSS version 22.0, cross checked for accuracy by two co-researchers and analyzed and interpreted by the Primary Investigator. For phase I study, an ICC test,percent agreement, and kappa statistic was used to analyze both inter-tester and intra-tester reliability. For phase II of the research study, a mixed design repeated measures ANOVA was used to analyze the differences from pretest to posttest in DRA measurements between each group over time, as well as to determine if there was a significant difference in ODI, PFDI, and pain scores after the completion of the six week exercise group. A Mann-Whitney $U$ was used to investigate the difference in DRA closure between women who participated in pre-natal exercise classes versus those who did not. A multiple linear regression and non-parametric Spearman Rho were used to determine if age, multi-birth, and previous exercise level correlated with DRA diameter in postpartum women. To determine if there was a difference between groups according to DRA chronicity and closure, a Kruskal-Wallis was calculated and analyzed.

\section{Results}

Prior to data collection, intra and inter-tester reliability was evaluated and revealed that both had high reliability for diagnostic ultrasound measurement at $r=0.945-0.989, p<0.005$. The research sample consisted of nine postpartum women who fit the eligibility requirements and voluntarily chose to participate in the study. However, only eight participated in both the pretest and posttest measurements. Demographics and baseline measurements are listed in Table 1 for both the traditional and experimental treatment groups.

The primary research question was to determine if there was a difference between the traditional exercise treatment and an experimental exercise treatment in DRA closure, pain, function, low back pain, and health-related quality of life. There was not a significant difference above the umbilicus ( $F=1.99$, 
Walton et al. Physical Therapy and Rehabilitation 2016,

http://www.hoajonline.com/journals/pdf/2055-2386-3-3.pdf

Table 1. Demographics and baseline measurements.

\begin{tabular}{lllllllll}
\hline Subject & Group & Age & BMI & $\begin{array}{l}\text { Delivery } \\
\text { Method }\end{array}$ & $\begin{array}{l}\text { DRA at } \\
\text { Umbilicus }\end{array}$ & ODI & PFDI & $\begin{array}{l}\text { Participation } \\
\text { in Pre-Natal } \\
\text { Exercises }\end{array}$ \\
\hline 1 & Experimental & 32 & 27.1 & Vaginal & $7.27 \mathrm{~mm}$ & 11 & 143.7 & No \\
2 & Experimental & 37 & 29.8 & Vaginal & $8.75 \mathrm{~mm}$ & 19 & 25 & Yes \\
3 & Experimental & 30 & 26.7 & Vaginal & $9.14 \mathrm{~mm}$ & 3 & 35.4 & Yes \\
4 & Traditional & 33 & 19.4 & Vaginal & $8.89 \mathrm{~mm}$ & 4 & 37.5 & Yes \\
5 & Traditional & 31 & 27.4 & Vaginal & $10.05 \mathrm{~mm}$ & 0 & 0 & Yes \\
6 & Experimental & 35 & 40.2 & Caesarean & $9.52 \mathrm{~mm}$ & 1 & 9.4 & No \\
7 & Experimental & 31 & 28.8 & Vaginal & $9.07 \mathrm{~mm}$ & 2 & 48.9 & No \\
8 & Traditional & 29 & 23.3 & Vaginal & $11.51 \mathrm{~mm}$ & 6 & 50 & No \\
9 & Traditional & 38 & 31.3 & Vaginal & $13.43 \mathrm{~mm}$ & 1 & 0 & No \\
\hline
\end{tabular}

$\mathrm{p}=0.208)$ and below the umbilicus $(\mathrm{F}=0.195, \mathrm{p}=0.674)$ (Figure 1$)$ by group for DRA closure; however, there was a significant difference after treatment at the level of the umbilicus ( $F=7.28$, $\mathrm{p}=0.036$ ), for both groups, with the traditional group showing greater closure after treatment $(M(p r e)=10.97+/-1.96$; $M($ post $)=6.63+/-1.65$ ) compared to the experimental group $(\mathrm{M}($ pre $)=8.75+/-0.87)(\mathrm{M}($ post $)=7.58+/-2.01)$. There was not a significant difference between pre and post-test scores on the PFDI questionnaire by group $(F=0.491 \mathrm{p}=0.510)$; however, significance was seen $(F=4.881, p=0.069)$ for the entire sample, with mean scores before treatment (Traditional Mean $=21.88+/-25.77$ and Experimental Mean $=52.48+/-53.00$ ). Individual sections of the PFDI, including the UDI, POPDI, and CRADI were also analyzed. A significant difference was not found for the UDI score ( $F=3.34, p=0.117)$, the POPDI score $(\mathrm{F}=0.017, \mathrm{p}=0.092)$, and the CRADI scores $(\mathrm{F}=0.387, \mathrm{p}=0.557)$ between groups. Furthermore, within group measurements did not show a significant difference between pre-test and post-test by function in terms of UDI scores $(Z=-1.59, p=0.111)$ and POPDI scores $(Z=-1.483, p=0.138)$. However, there was a significant difference for the CRADI scores $(Z=-2.032, p=0.042)$ within both groups (Figure 2). A significant difference was

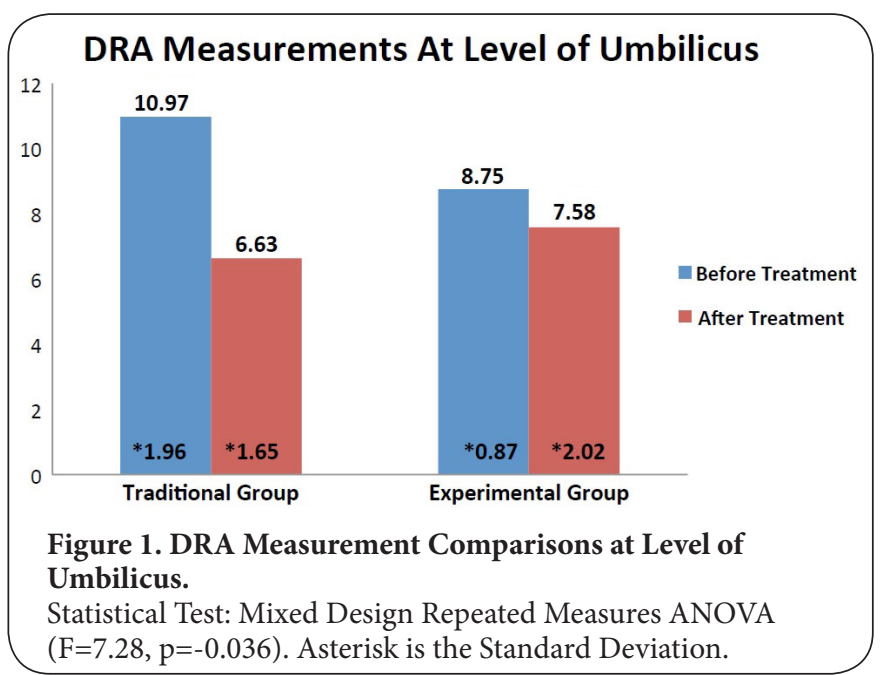

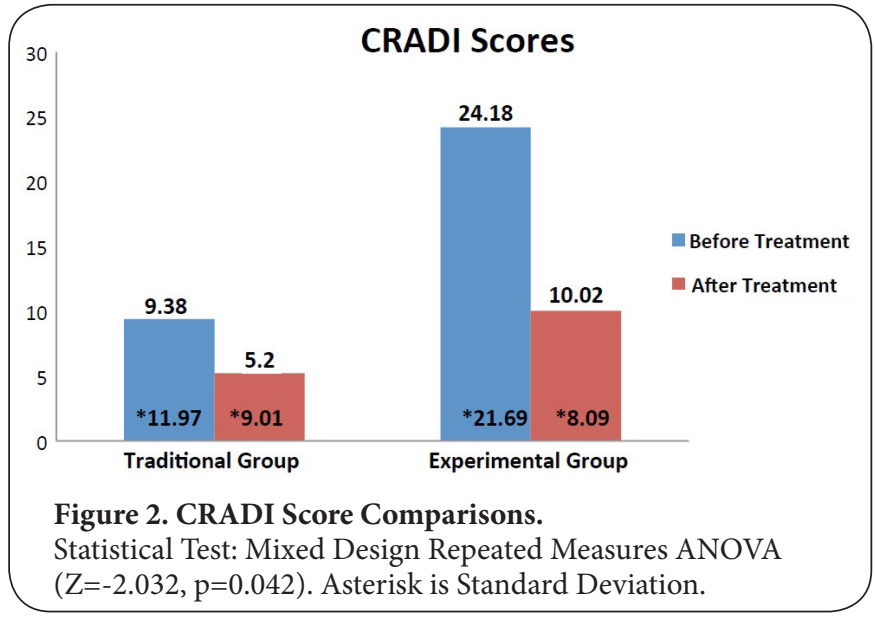

not found between pre to post-test for the variables, health related quality of life and pain scores via the ODI for the overall sample ( $F=2.64, p=0.155)$, between groups $(F=0.362, p=0.569)$ and within individual treatment groups $(\mathrm{F}=0.886, \mathrm{p}=0.383)$. The ODI scores for both the traditional (M(pre): $2.75+/-2.75$ ) (M(post): $2.33+/-1.15$ ) and experimental (M(pre): $7.20+/-7.69)$ (M(post): $2.20+/-1.79$ ) groups decreased from pre to post test significantly meaning there was not a significant difference between the groups. However, there was not a significant difference between the groups, although the experimental group showed greater improvement overall (Figure 3).

The first secondary question was to determine if there was a difference between DRA measurements in relation to the women who participated in pre-natal exercises. A significant difference was not found between any of the DRA measurements at all three locations, with respect to those who participated in pre-natal exercises and those who did not report participation prior to treatment (above umbilicus: $Z=-0.980$, $p=0.327$ ), (at umbilicus: $Z=-0.735, p=0.462$ ), and (below umbilicus: $Z=-0.980, p=0.327)$. The secondary research question was to determine if scores from ODI and PFDI, parity, age, and participation in pre-natal exercises were able to predict baseline DRA measurements. No predictive relationship was 


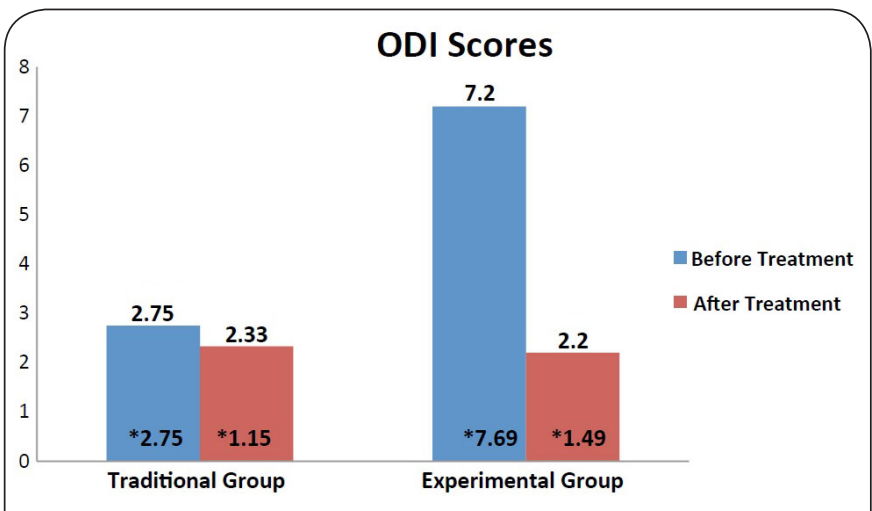

Figure 3. ODI Score Comparisons.

Statistical Test: Mixed Repeated Measures ANOVA; overall sample $(\mathrm{F}=2.64, \mathrm{p}=0.155)$, between groups $(\mathrm{F}=0.362, \mathrm{p}=0.569)$ and within individual treatment groups $(\mathrm{F}=0.886, \mathrm{p}=0.383)$ Asterisk is Standard Deviation.

found with respect to ODI, PFDI, age, parity, previous levels of exercise and prediction of DRA measurements, $(r=0.771$, $\left.r^{2}=0.594, p=0.582\right)$, indicating no significant prediction. There was also a non-significant correlation between PFDI scores and DRA measurements $\left(r=0.605, r^{2}=0.366, p=0.084\right)$. The third secondary question was to determine if there was a difference between chronicity and DRA closure. There was not a significant difference between chronicity and DRA closure (Chi-square $=3.472, p=0.482$ ). The average closure measurement for the entire sample at the level of the umbilicus was 2.48 millimeters and the average chronicity of the sample was 6 months to 1 year.

\section{Discussion}

DRA is a common health condition that occurs during pregnancy and postpartum periods, and if not corrected could produce a number of health complications including low back pain, reduced function, and quality of life. The purpose of this study was to determine if there was a significant difference between a six week experimental core stability plank exercise program compared to a traditional supine exercise program in the closure of DRA, and to determine the relationship between DRA measurements, pain, ODI, and PFDI scores. This research study found that following either exercise program for six weeks resulted in a significant difference at the level of the umbilicus by group for DRA closure. However, there was not a significant DRA closure above or below the umbilicus. This has been found to be significant because the "umbilicus is the most common site for DRA. Forty seven percent (47\%) of the measurements at the umbilicus show a DRA" [4], according to a study conducted by Parker et al (2009).

Although this study did not find significant closure above or below the umbilicus, it does suggest that a core exercise program in addition to abdominal bracing during exercise, using either program, could be a successful option for DRA treatment. In addition, other studies have found exercises to be useful in the closure of DRA. For instance, a case study conducted by Litos (2014) on the therapeutic effects of exercise on DRA closure found not only increased abdominal strength and endurance, but also a reduction of two centimeters in DRA following stabilization exercises and use of abdominal bracing [19]. Unlike this research study, the participant in Litos's study presented with a severe DRA reaching, "a total length of more than $9 \mathrm{~cm}$ long" [19]. The same study was also conducted for four months, and it included a combination of patient education, core stabilization exercises consisting of pelvic floor muscle "holds," supine marching, supine straight leg raise, bridges, posterior pelvic tilts, clamshells, modified planks, lunges, and crunches among other dynamic and hip strengthening exercises, along with the use of an abdominal belt throughout the day. Although this research study was only six weeks long, included a total of four exercises, and consisted of less severe cases of DRA, it too revealed positive outcomes and DRA closure following exercise programs using abdominal bracing. In addition, these exercise programs were more time efficient to administer, did not require abdominal bracing the entire day, and showed DRA closure in less severe cases, making it more feasible to complete, and appealing for clients and clinicians.

A significant difference was found, from pre-test to post-test measurement, in PFDI questionnaire, with respect to CRADI scores for both groups. However, the experimental group mean reported higher reduction in CRADI scores than the traditional group mean. However, there was not a significant difference for the entire sample or within group scores for the overall PFDI scores, UDI and POPDI. Participants in this study began with higher reported levels of distress in CRADI scores than any of the other sections. The significant drop in scores in CRADI but not in other scores may be attributed to the effects of both exercise groups on reducing the colorectal-anal symptom (CRADI) scores. Future research should address this issue with a sample of subjects reporting higher baseline urinary and pelvic distress symptoms to further explore this finding.

Although there was not a significant difference for health related quality of life and pain changes, using the ODI, both groups demonstrated decreased scores, with the experimental showing greater decrease than the traditional group. The trend toward reduction in both scores may indicate that both the experimental and traditional group benefitted from the treatment, with both groups reporting higher mental health related quality of life and decreased pain, following treatment. This study's experimental group initially presented with a mean ODI score of 7.20, while the control group had a low 2.75. Both groups were categorized as displaying minimal disability according to the ODI. Further research using a greater sample size and a sample with reports of greater degree of disability may provide more illumination on the effects regarding health related quality of life and pain scores. 
Furthermore, this study did not find a difference in DRA measurements, at any of the three locations, for women who participated in pre-natal exercise classes compared to those that did not at all three levels. Yet, a study conducted by Chiarello et al., (2005) which included eight pregnant women who had participated in a six week prenatal class and ten women who did not report participation in exercise, found a significant difference between the effects of exercise and DRA in the pre-natal exercise group $(p=0.0009)$, although it did not find a significant difference between the number of pregnancies each women had in relation to the presence of DRA $(p=0.611)[6]$. The prenatal exercise program in the same study consisted of posterior pelvic tilts, transverse abdominis contraction head lifts with kegels, military press, lateral raises, chest press, shoulder external rotation, bicep curls, scapular retraction, triceps extension, forward pull, overhead pull sitting, bridges, hip flexion, abduction, and adduction all with exercise bands. It found that, post-partum DRA measurements were up to $52.2 \%$ higher in the non exercise group with a "DRA of $59.5 \mathrm{~mm}$ at the umbilicus", than the exercise group with a measurement of $11.4 \mathrm{~mm}$ [6]. Overall, it found that pregnant women who did not participate in the exercise program exhibited a statistically larger separation of the rectus abdominis regardless of the location of measurement and the number of previous pregnancies. The differences in our research and prior research results may be attributed to the prospective nature of Chiarello's study compared to the retrospective baseline measurements of our study.

Non-significant correlations were found between the overall PFDI and DRA, ODI and DRA, and the DRA with respect to age, parity and previous exercise levels. However, trends toward decreases in pain and disability with respect to DRA closure were reported for our sample. Furthermore, DRA may complicate the pelvic floor by placing undue stress and emphasis on the role of support for the abdominal and pelvic cavity, causing acute symptoms of hypertonicity of the pelvic floor. This excessive stress may, in time, produce symptoms related to urinary and pelvic distress, but were missed in the small postpartum sample of this study. Finally, due to the lack of evidence in the literature on the effects of chronicity on DRA closure, the researchers sought to assess if there was a correlation between DRA diameter and chronicity of the postpartum period by comparing baseline measurements across the sample. No significant correlation was found between DRA and postpartum chronicity.

\section{Delimitations of this study}

To create clear boundaries for this study, inclusion and exclusion criteria were developed. The inclusion criteria helped the researchers identify individuals who could participate in the study, by clearly stating the condition necessary to participate, the appropriate timing of the pregnancies, and the age of individuals that could participate. The exclusion criteria aided the researchers in determining who should not participate in the study, by listing conditions that could be negatively affected by the exercises prescribed. Overall, the inclusion and exclusion criteria were important factors for the homogeneity of the research participants.

To increase internal validity of the study the researchers considered and carried out the following: Each subject was asked to complete a health questionnaire before data collection. If the subject stated they participated in a pre-natal exercise class, this information was taken into account when processing the statistical analysis of the data.

The researchers attempted to reducesubject attrition by contacting each subject on the second, fourth, and sixth week to assess how the home exercise program was progressing and ask the subjects if they had any questions or comments about the exercises. The week before posttest measurements were taken, the researchers contacted each participant to encourage full participation of posttest measurements.

The researchers accounted for testing and instrumentation validity by completing measurements using the same caliper and diagnostic ultrasound machine for both pretest and posttest measurements. The machine was calibrated to the same settings, both measurements were taken in the same environment, and by the same researchers.

The external validity of this study was improved by adhering to the outline as follows:

To decrease bias, two different researchers performed the DRA measurements with both the calipers and ultrasound without knowing what measurements the other person found. As an attempt to decrease measurement error a pilot study for inter-rater and intra-rater reliability for the use of calipers was completed, then compared to ultrasound measurements prior to the study to ensure the measurement tools were used appropriately. Prior to measurements, the US machine was checked for calibration, and calipers were zeroed out to reduce bias. The researchers found strong intra-rater and inter-rater reliability for the diagnostic ultrasound measurements $(r=0.945-0.989, p<0.0005)$. In an attempt to limit bias between researchers and subjects a specific script was read to every subject before the beginning of participation in the research study.

Finally, in order to limit bias that could occur from the environment, all measurements were taken at the same location, on the same type of surface for both pre and postmeasurements of DRA by the same co-researchers.

\section{Clinical application}

The results of this study indicate that both strengthening programs produced significant closure from pre to post-test, with the traditional exercise group showing a slightly greater improvement in DRA closure over time. This information demonstrates that a physical therapist can design and utilize either a traditional abdominal crunch exercise program or a plank-based exercise program. Both exercise programs were found to be effective for closure of DRA and reduced 
Walton et al. Physical Therapy and Rehabilitation 2016,

colo-rectal-anal symptoms (CRADI) of pain and dysfunction when coupled with the stabilization belt during exercise. These exercises should be completed at home, three times a week, for a total of six weeks for postpartum women diagnosed with DRA.

\section{Limitations}

Limitations of this current study include comparing the experimental group to a traditional treatment group rather than a true control group. Lack of previous research using plank exercises as a treatment option for DRA made appropriate dosage very difficult. The small sample size limited generalizability of this research. Other limitations include missing data for exercises logs, reports of inconsistent exercise by some subjects, the fact that most participants gave birth vaginally,variations in abdominal brace position, and relatively short exercise time. These limitations call for a more controlled exercise setting, and a stratified purposive random sampling of groups by birth mode and parity for future research in this area.

\section{Future research}

Future research should include also use of a true control group to increase the comparison validity of the study, a larger sample size to increase generalizability of the study, and research by birth mode and parity to see if birth mode or parity has an effect on exercise results. Future research should identify specific exercise dosage of plank exercises for the treatment of DRA. Increasing the length of the study, giving a standardized brace to each participant, and more supervised exercise sessions with objective recording of all exercises prescribed are suggested for future research.

\section{Conclusion}

The purpose of this randomized controlled study was to determine if there was a significant difference between a six week experimental core stability plank program compared to traditional supine exercise program in the closure of DRA. Additionally, this study sought to determine the relationship between DRA measurements, pain, ODI, and PFDI scores. Overall, There was not a significant difference between groups with respect to DRA closure. However, in both the experimental group and traditional exercise group there was a significant decline in the DRA measurement at the umbilicus $(F=7.28, p=0.036)$, with the traditional group showing a greater decline from pre to posttest $(M($ pre $)=10.97+/-1.96$; $M($ post $)=6.63+/-1.65$ ) compared to the experimental group $(\mathrm{M}($ pre $)=8.75+/-0.87)(\mathrm{M}($ post $)=7.58+/-2.01)$. This study also showed a significant improvement in CRADI scores for both groups from pre-test to post-test, suggesting either exercise program to be valuable in treatment of women with colorectal-anal symptoms in the postpartum period.

\section{Competing interests}

The authors declare that they have no competing interests.

Authors' contributions
\begin{tabular}{|l|c|c|c|c|c|}
\hline Authors' contributions & LMW & DB & AC & SM & BS \\
\hline Research concept and design & $\checkmark$ & -- & -- & -- & -- \\
\hline Collection and/or assembly of data & $\checkmark$ & $\checkmark$ & $\checkmark$ & $\checkmark$ & $\checkmark$ \\
\hline Data analysis and interpretation & $\checkmark$ & -- & -- & -- & -- \\
\hline Writing the article & $\checkmark$ & $\checkmark$ & $\checkmark$ & $\checkmark$ & $\checkmark$ \\
\hline Critical revision of the article & $\checkmark$ & -- & -- & -- & -- \\
\hline Final approval of article & $\checkmark$ & -- & -- & -- & -- \\
\hline Statistical analysis & $\checkmark$ & $\checkmark$ & $\checkmark$ & $\checkmark$ & $\checkmark$ \\
\hline
\end{tabular}

Acknowledgement

A special thank you to each of the women who voluntarily participated in this research study.

Publication history

Editor: Gordon John Alderink, Grand Valley State University, USA. Received: 22-Jan-2016 Final Revised: 14-Mar-2016

Accepted: 04-Apr-2016 Published: 11-Apr-2016

\section{References}

1. Benjamin DR, van de Water AT and Peiris CL. Effects of exercise on diastasis of the rectus abdominis muscle in the antenatal and postnatal periods: a systematic review. Physiotherapy. 2014; 100:1-8. | Article | PubMed Abstract | PubMed FullText

2. Mota P, Pascoal AG, Sancho F and Bo K. Test-retest and intrarater reliability of 2-dimensional ultrasound measurements of distance between rectus abdominis in women. J Orthop Sports Phys Ther. 2012; 42:940-6. | Article | PubMed

3. Beer GM, Schuster A, Seifert B, Manestar M, Mihic-Probst D and Weber SA. The normal width of the linea alba in nulliparous women. Clin Anat. 2009; 22:706-11. | Article | PubMed

4. Parker M, Millar L and Dugan S. Diastasis Rectus Abdominis and LumboPelvic Pain and Dysfunction--Are They Related? Journal of Women's Health Physical Therapy. 2009; 33:15-22.

5. Mogren IM. Physical activity and persistent low back pain and pelvic pain post partum. BMC Public Health. 2008; 8:417. | Article I PubMed Abstract | PubMed FullText

6. Chiarello C, Falzone L, McCaslin K, Patel M and Ulery K. The effects of an exercise program on diastasis recti abdominis in pregnant women. Journal Of Women's Health Physical Therapy. 2005; 29:11-16.

7. Pascoal AG, Dionisio S, Cordeiro F and Mota P. Inter-rectus distance in postpartum women can be reduced by isometric contraction of the abdominal muscles: a preliminary case-control study. Physiotherapy. 2014; 100:344-8. | Article | PubMed

8. El-Mekawy H, Eldeeb A, El- Lythy M and El-Begawy A. Effect of Abdominal Exercises versus Abdominal Supporting Belt on Post-Partum Abdominal Efficiency and Rectus Separation. International Journal of Medical, Health, Pharmaceutical and Biomedical Engineering. 2013; 7:4448.

9. Hsia $\mathrm{M}$ and Jones $\mathrm{S}$. Natural resolution of rectus abdominis diastasis. Two single case studies. Aust J Physiother. 2000; 46:301-307. I Article I PubMed

10. Gilleard WL and Brown JM. Structure and function of the abdominal muscles in primigravid subjects during pregnancy and the immediate postbirth period. Phys Ther. 1996; 76:750-62. | Article | PubMed

11. Mendes Dde A, Nahas FX, Veiga DF, Mendes FV, Figueiras RG, Gomes HC, Ely PB, Novo NF and Ferreira LM. Ultrasonography for measuring rectus abdominis muscles diastasis. Acta Cir Bras. 2007; 22:182-6. | Article | PubMed

12. Barbosa S, de Sa RA and Coca Velarde LG. Diastasis of rectus abdominis in the immediate puerperium: correlation between imaging diagnosis 
Walton et al. Physical Therapy and Rehabilitation 2016,

http://www.hoajonline.com/journals/pdf/2055-2386-3-3.pdf

and clinical examination. Arch Gynecol Obstet. 2013; 288:299-303. | Article | PubMed

13. Chiarello CM and McAuley JA. Concurrent validity of calipers and ultrasound imaging to measure interrecti distance. J Orthop Sports Phys Ther. 2013; 43:495-503. | Article | PubMed

14. Smeets R, Koke A, Lin CW, Ferreira M and Demoulin C. Measures of function in low back pain/disorders: Low Back Pain Rating Scale (LBPRS), Oswestry Disability Index (ODI), Progressive Isoinertial Lifting Evaluation (PILE), Quebec Back Pain Disability Scale (QBPDS), and Roland-Morris Disability Questionnaire (RDQ). Arthritis Care Res (Hoboken). 2011; 63 Suppl 11:S158-73. | Article | PubMed

15. Davidson $\mathrm{M}$ and Keating JL. A comparison of five low back disability questionnaires: reliability and responsiveness. Phys Ther. 2002; 82:8-24. | Article | PubMed

16. Hawker G, Mian S, Kendzerska T and French M. Measures of Adult Pain. Arthritis Care \& Research. 2011; 63:240-252.

17. Geller EJ, Barbee ER, Wu JM, Loomis MJ and Visco AG. Validation of telephone administration of 2 condition-specific quality-of-life questionnaires. Am J Obstet Gynecol. 2007; 197:632 e1-4. | Article | PubMed

18. Barber MD, Walters MD and Bump RC. Short forms of two conditionspecific quality-of-life questionnaires for women with pelvic floor disorders (PFDI-20 and PFIQ-7). Am J Obstet Gynecol. 2005; 193:103-13. | Article | PubMed

19. Litos K. Progressive Therapeutic Exercise Program for Successful Treatment of a Postpartum Woman With a Severe Diastasis Recti Abdominis. Journal Of Women's Health Physical Therapy. 2014; 38:5873. | Article

20. Fairbank JC and Pynsent PB. The Oswestry Disability Index. Spine (Phila Pa 1976). 2000; 25:2940-52. | Article | PubMed

21. Mota P, Pascoal AG, Sancho F, Carita Al and Bo K. Reliability of the inter-rectus distance measured by palpation. Comparison of palpation and ultrasound measurements. Man Ther. 2013; 18:294-8. | Article | PubMed

22. To WW and Wong MW. Factors associated with back pain symptoms in pregnancy and the persistence of pain 2 years after pregnancy. Acta Obstet Gynecol Scand. 2003; 82:1086-91. | Article | PubMed

\section{Citation:}

Walton LM, Costa A, LaVanture D, McIlrath S and Stebbins $B$. The effects of a 6 week dynamic core stability plank exercise program compared to a traditional supine core stability strengthening program on diastasis recti abdominis closure, pain, oswestry disability index (ODI) and pelvic floor disability index scores (PFDI). Phys Ther Rehabil. 2016; 3:3.

http://dx.doi.org/10.7243/2055-2386-3-3 